\title{
Searching for correlations in Gaia DR2 unbound star trajectories
}

\author{
F. Montanari ${ }^{1 \star}$, D. Barrado ${ }^{2} \dagger$, and J. García-Bellido ${ }^{\ddagger}$ \\ ${ }^{1}$ Instituto de Física Teórica IFT-UAM/CSIC, Universidad Autónoma de Madrid, Cantoblanco 28049 Madrid, Spain \\ ${ }^{2}$ Departamento de Astrofísica, Centro de Astrobiología (CSIC-INTA), ESA-ESAC. Camino Bajo del Castillo s/n. \\ 28692 Villanueva de la Cañada, Madrid, Spain
}

18 November 2019

\begin{abstract}
Scattering events with compact objects are expected in the primordial black hole (PBH) cold dark matter (CDM) scenario due to close encounters between stars and PBH in the dense environments of dwarf spheroidals. We develop a Bayesian framework to search for correlations among Milky Way stellar trajectories and those of globular clusters and dwarf galaxies in the halo, and other nearby galaxies. We apply the method to a selection of hypervelocity stars (HVS) and globular clusters from Gaia DR2 catalog, and known nearby (mostly dwarf) galaxies with full phase-space and size measurements. We report positive evidence for trajectory intersection 20-40 Myr ago of up to 2 stars, depending on priors, with the Sagittarius dwarf Spheroidal (dSph) galaxy when assuming Marchetti et al. (2019) distance estimates. We verify that the result is compatible with their evolutionary status, setting a lower bound for the stellar age of 100 Myr. However, such scattering events are not confirmed when assuming Anders et al. (2019) distance estimates. We discuss shortcomings related to present data quality and future prospects for detection of HVS with the full Gaia catalog and Sagittarius dSph.
\end{abstract}

Key words: cosmology: dark matter - Galaxy: kinematics and dynamics - Galaxy: stellar content - galaxies: kinematics and dynamics - galaxies: statistics

\section{INTRODUCTION}

The hierarchical structure formation scenario assumes that large galaxies are formed by mergers of smaller ones, which bring in both gas (hydrogen), stars and dark matter (DM). These smaller structures, generically called dwarf galaxies, orbit around the larger galaxy and interact with it. Some appear tidally disrupted by previous crossings through the disk and are elongated, and others are still approaching it and have more or less spherical shape. All this substructure have large mass-to-light ratios, in some cases larger than 1000 , making them extremely difficult to detect in the sky. Their numbers were predicted, within the cold dark matter scenario, to be large, hundreds to thousands of objects orbiting each large galaxy. However, only about a dozen had been observed until SDSS and DES discovered several tens of them (Drlica-Wagner et al. 2015; Newton et al. 2018; Simon et al. 2019), solving the so-called substructure problem when extrapolated to the whole sky.

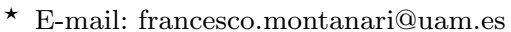

$\dagger$ E-mail: barrado@cab.inta-csic.es

† E-mail: juan.garciabellido@uam.es
}

The low surface brightness of dwarf galaxies could be explained in the PBH CDM scenario (García-Bellido 2017) due to the loss of stars via close encounters with massive primordial black holes comprising the dark matter halos of all galaxies. In this scenario, stars in the shallow potential wells of dwarf galaxies are more likely to get slingshot away due to close encounters with DM black holes, acquiring velocities in the hundreds to thousands of $\mathrm{km} / \mathrm{s}$ (Clesse \& García-Bellido 2017). Such hypervelocity stars (HVS), likely unbound to the Milky Way potential, should travel across the sky and their trajectories should point back to the dSph from which they originate. High-velocity stars are also found in the core of globular clusters (GC) (Lützgendorf et al. 2012), which may indicate a population of $\mathrm{PBH}$, and also in this case some of them may acquire a velocity above the escape threshold (Clesse \& García-Bellido 2017).

In this paper we develop a Bayesian framework for the detection of such close encounters via the correlation of stellar trajectories in Gaia DR2 catalog (Gaia Collaboration et al. 2016, 2018a) with trajectories of dwarf and other nearby galaxies, and GC. If massive black holes are indeed responsible for the depletion of stars from dwarf galaxies, it 
is expected that a few slingshot events must have happened in the last 100 million years inside dwarf galaxies in the Milky Way. In particular, since the probability of events is proportional to the density of the dwarf galaxy, one expects the most massive ones to be the source of HVS. Unfortunately, Gaia has limited resolution for distant stars, and only those relatively close to the Sun are measured with sufficient accuracy in $6 \mathrm{D}$ phase space (we consider stars up to $\sim 13 \mathrm{kpc}$ ).

Recently, Marchetti et al. (2019) found that some of the observed HVS were not pointing away from the center of the Milky Way, as was naively expected, but rather towards the disk, as if they had originated in the halo of our galaxy. Furthermore, Hattori et al. (2018) reported one star whose orbit has non-negligible probability of having passed near the Large Magellanic Cloud in the past. This prompted us to explore the possible origin of HVS and whether they could originate in the $\mathrm{dSph}$ that orbit around the MW within a radius of several tens of kiloparsecs, and which could have been travelling for several tens of millions of years from their sources and velocities up to ten times larger than typical stellar velocities in the halo.

We compute the close encounter evidence between HVS and Milky Way GC, dwarf, and nearby galaxies studying the posterior distribution of an impact parameter defined upon phase-space and size information. The evolutionary status of HVS scattering candidates is further analyzed based to their Hertzsprung-Russell (HR) diagram to confirm that their expected age is consistent with having travelled over typically large distances.

In section 2 we illustrate our Gaia DR2 HVS catalog, and the data selection for Milky Way GC and nearby galaxies discussing distribution and kinematic properties properties of each selection. In section 3 we outline our Bayesian methodology to evaluate the scattering evidence between HVS and compact objects in GC or nearby galaxies. In section 4 we give our results. We conclude in section 5 . In appendix A we discuss results based on an alternative stellar selection than the one considered throughout the main paper. In appendix B we define our Galactocentric reference frame. In appendix $\mathrm{C}$ we provide references for the selected GC and galaxies, as well as orbit data for Sagittarius dSph and HVS compatible with having crossed its trajectory.

\section{DATA}

\subsection{Hyper-velocity stars}

Our reference catalog is Gaia DR2 (Gaia Collaboration et al. 2016, 2018a), an all sky survey consiting of more than 1.3 billion stars. It contains accurate accurate positions $(\alpha, \delta)$, proper motions $\left(\mu_{\alpha *}, \mu_{\delta}\right)$, parallax $(\omega)$, radial velocity, magnitudes and colors for the bright end, for $\sim 7$ million stars. We base our analysis on the 7183262 stars selection provided by Marchetti et al. (2019), established with the following quality cuts (see also Gaia DR2 documentation ${ }^{1}$ for more information about variables description):

- astrometric_gof_al $<3$.

\footnotetext{
1 https://gea.esac.esa.int/archive/documentation/GDR2/
}

- astrometric_excess_noise_sig $\leq 2$.

- $-0.23 \leq$ mean_varpi_factor_al $\leq 0.32$.

- visibility_periods_used $>8$.

- rv_nb_transits $>5$.

We further clean the sample:

- Selecting heliocentric total velocities in the Galactic rest frame large enough compared to their uncertainties $v-\sigma_{v} \gtrsim 500 \mathrm{~km} / \mathrm{s}$, to mitigate errors in the Galactic absolute velocity.

- Removing potentially spurious radial velocities (Boubert et al. 2019). ${ }^{2}$

Marchetti et al. (2019) provides for each star the probability $P_{\mathrm{ub}}$ of being unbound to the Milky Way potential. Our final selection of 1649 stars takes into account this information and further photometric and astrometric quality cuts (Schönrich et al. 2019):

- $P_{\mathrm{ub}}>0.5$.

- Color cut $G_{\mathrm{BP}}-G_{\mathrm{RP}}<1.5 \mathrm{mag}$.

- Magnitude cut $G<14.5 \mathrm{mag}$, and $G, G_{\mathrm{BP}}, G_{\mathrm{RP}}>0$ mag.

- BP-RP excess flux factor cut $1.172<E_{\text {bprp }}<1.3$.

- astrometric_excess_noise $<1$.

See appendix A for an alternative stellar selection.

We consider distance estimates given in Marchetti et al. (2019). The catalog does not include systematic errors on parallax measurements, and it sets a large scale length in the distance prior for likely distant stars that may overestimate distances. To overcome these shortcomings, we also consider the more recent Anders et al. (2019) work that improves the accuracy of extinction and effective temperature estimates provided with Gaia DR2 by combining its astrometric and photometric measurements with external photometric catalogs. In particular, the related distance catalog includes errors induced by a parallax zero-point offset. We find that Marchetti et al. (2019) distances are systematically larger on average by roughly a factor of 2 than Anders et al. (2019) estimates for distant stars. The disagreement increases even up to an order of magnitude for stars whose distance is estimated to be $\lesssim 2 \mathrm{kpc}$ by Anders et al. (2019), whereas this latter catalog agrees well with other computations (Bailer-Jones et al. 2018; Schönrich et al. 2019) within this range. Probabilities $P_{\mathrm{ub}}$ used for our selection rely on Marchetti et al. (2019) distances, but evaluating $P_{\mathrm{ub}}$ based on Anders et al. (2019) is beyond the scope of the present work. Since $P_{\mathrm{ub}}$ does not enter in following computations, comparing results based on the two different distance estimates still provides a consistency check.

Figure 1 shows that most of our HVS selection is characterized by total Galactocentric velocities $V_{\text {tot }} \sim O\left(10^{3}\right) \mathrm{km} / \mathrm{s}$. For details about our coordinates system see appendix B. Figure 2 shows the Toomre diagram with the Galactocentric Cartesian $V_{y}$ component on the abscissa, and the $\sqrt{V_{x}^{2}+V_{z}^{2}}$ component on the ordinate. ${ }^{3}$ Here and afterwards error bars

2 The list of possibly contaminated radial velocities is available as ancillary file to arXiv:1901.10460 [astro-ph.SR].

3 The Toomre diagram is often expressed in terms of Galactic (heliocentric) Cartesian velocities U, V, W (e.g., Schönrich 2012) well suited to describe the solar neighborhood. Here we are in- 


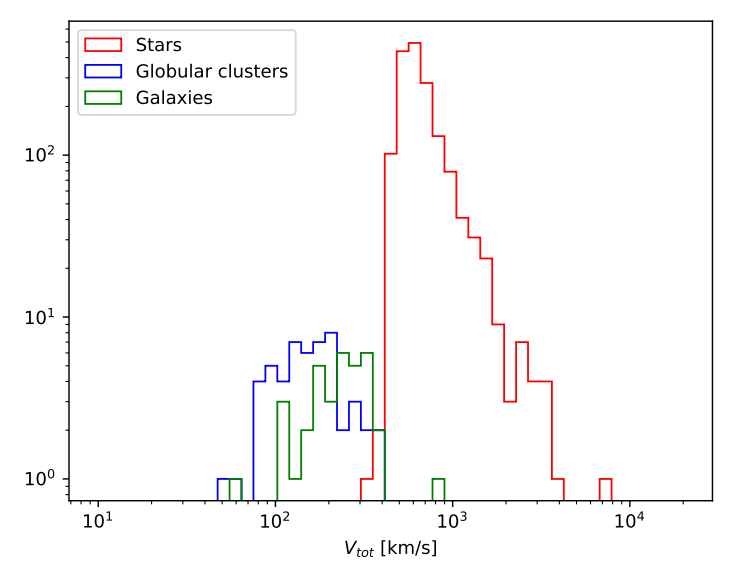

Figure 1. Total velocities in Galactocentric coordinates for our selection of stars, globular clusters and galaxies.

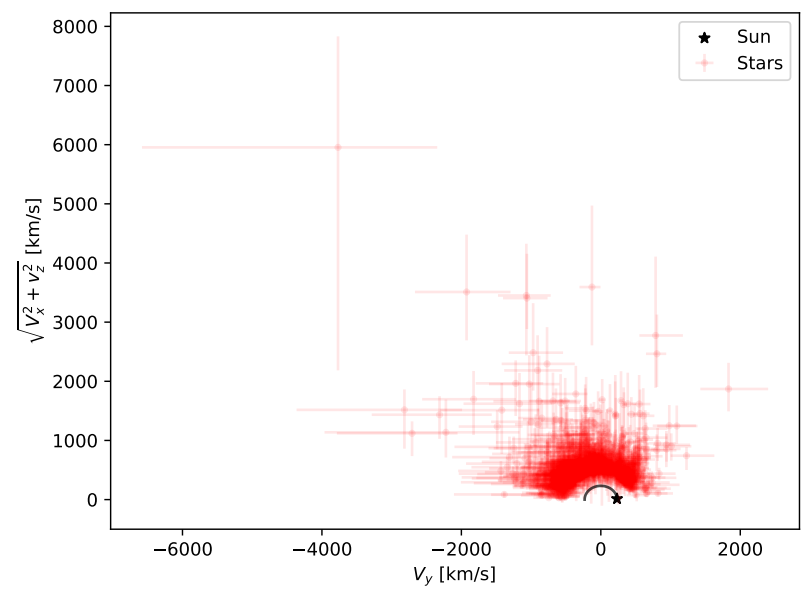

Figure 2. Toomre diagram in terms of Galactocentric Cartesian velocities for our HVS selection. All HVS are above the black semi-circle centered at the origin and of radius given by the Sun (black star) $V_{y}$ component.

indicates $68 \%$ confidence intervals. The diagram is populated only above a semi-circle centered at the origin and of radius given by the Sun $V_{y}$ component, suggesting that we select a population of halo stars (e.g., Bonaca et al. 2017). Figure 3 shows Galactocentric positions with error bars dominated by uncertainties on distances from the Sun.

\subsection{Globular clusters and galaxies}

We select 52 globular clusters (GC) identified in the Gaia DR2 catalog (Gaia Collaboration et al. 2018b) that report both full phase-space and radius, defined as the maximum radius at which proper-motion members are found.

terested in the dynamics of the Galaxy on a global scale and a Galactocentric frame is more convenient.
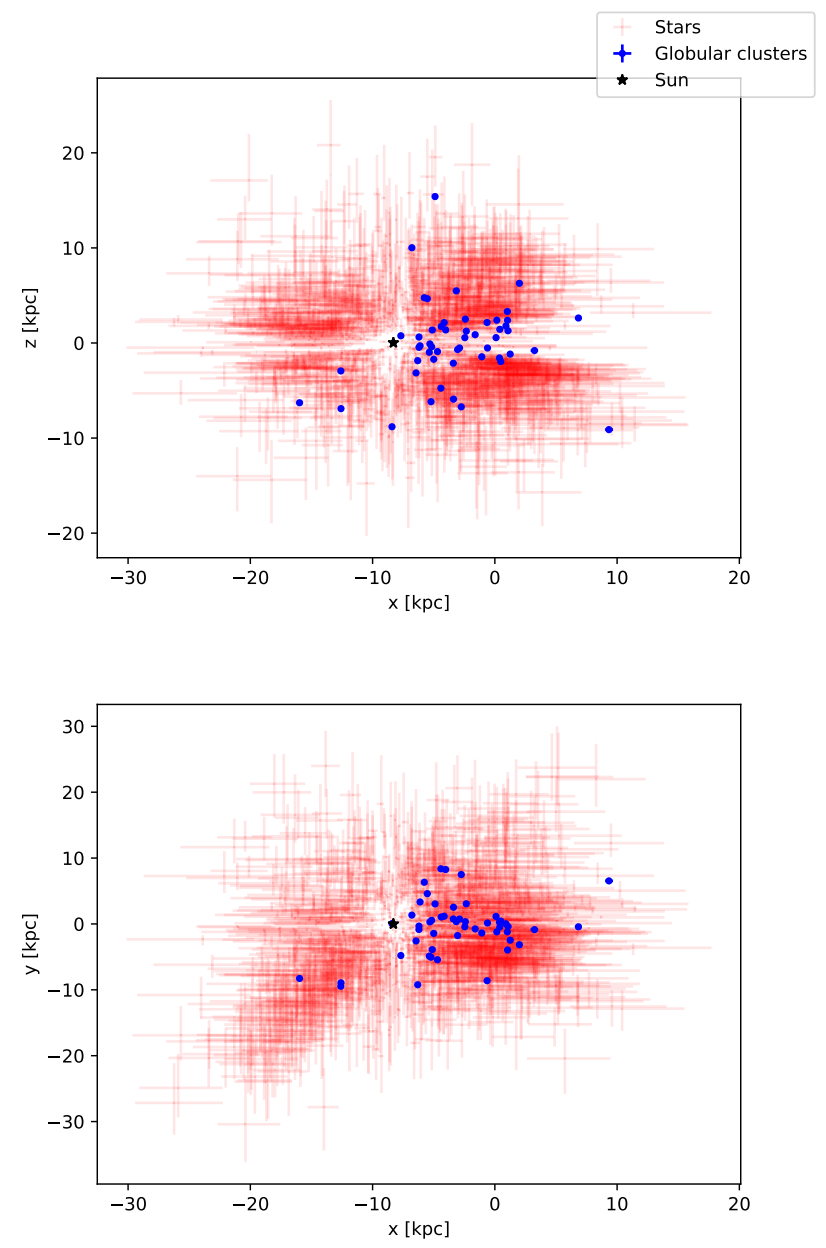

Figure 3. Galactocentric positions for stars and globular clusters. Error bars $\lesssim 3 \%$ for GC are smaller than markers (the latter are not representative of objects extension).

We select all galaxies for which we can match position and half-light radius (measured along major axes) given by ${ }^{4}$ McConnachie (2012) with peculiar motions of identified dwarf Milky Way satellite galaxies in Gaia DR2 (Fritz et al. 2018). We also include Antlia II (Torrealba et al. 2019) and Andromeda (velocity from van der Marel et al. 2012). ${ }^{5}$ In the latter case we retrieve the optical major-axis from the SIMBAD database (Wenger et al. 2000), also used to get sizes and velocities of the large (LMC) and small (SMC) Magellanic clouds that we combine with positions listed in McConnachie (2012). This gives a total of 35 galaxies.

Figure 1 shows total Galactocentric velocities $V_{\text {tot }} \sim$ $O\left(10^{2}\right) \mathrm{km} / \mathrm{s}$ both for GC and galaxies. Figures 3 and 4 show $\mathrm{GC}$ and galaxy positions, respectively. We verified that the asymmetric GC distribution, concentrated between the Sun

${ }^{4}$ We use the table updated on 20 September 2015 available at http://www.astro.uvic.ca/ alan/Nearby_Dwarf_Database. html.

5 van der Marel et al. (2012) assumes $V_{y}=239 \pm 5 \mathrm{~km} / \mathrm{s}$ for the Sun Galactocentric velocity component along the direction of Galactic rotation, while here we assume $232.24 \mathrm{~km} / \mathrm{s}$ (see appendix B). The difference is negligible for our purposes. 

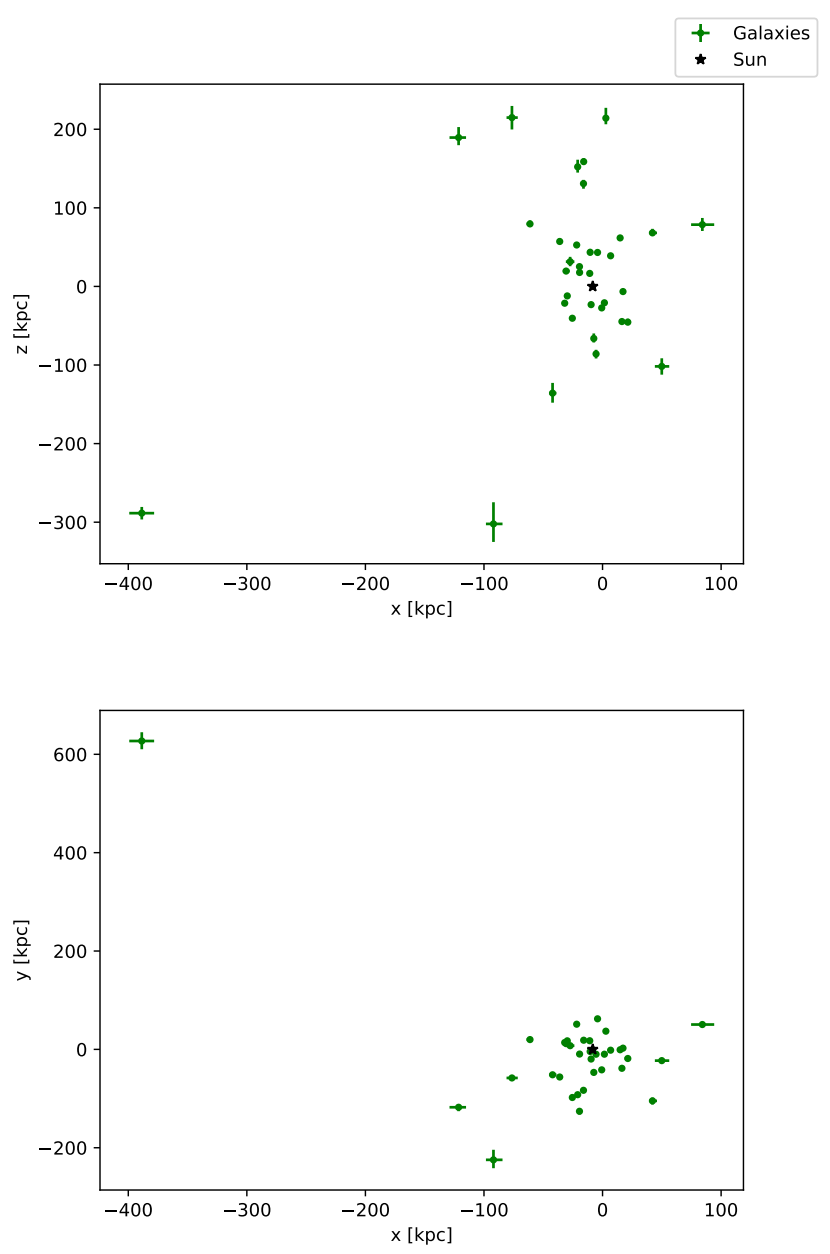

Figure 4. Galactocentric positions for galaxies. The further galaxy is Andromeda. Marker size (for some object larger that error bars) is not representative of object extension.

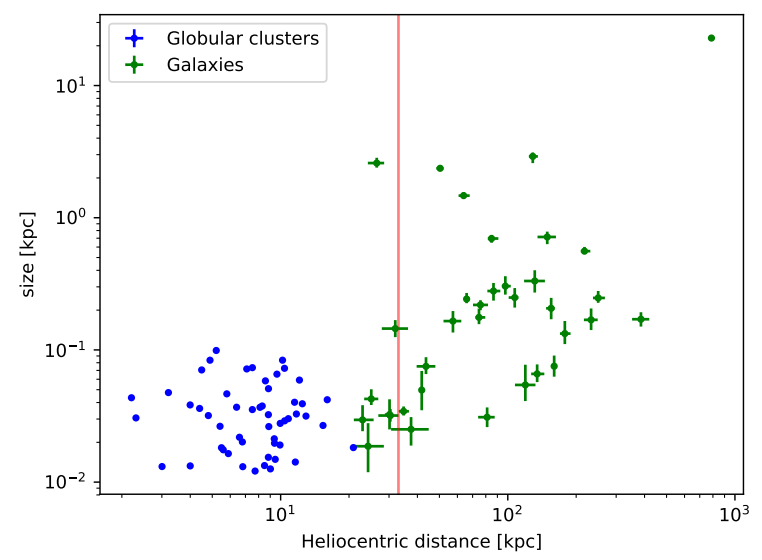

Figure 5. Heliocentric distance and size for GC and galaxies. The vertical line corresponds to the further HVS; the largest object within this distance is Sagittarius $\mathrm{dSph}$, placed at coordinates $(26 \pm 2,2.6 \pm 0.2) \mathrm{kpc}$. and the galactic Center is also present in the full catalog and not only in our selection (note the lack of GC in the upper quadrants for objects with $x<x_{\odot} \approx-8.3 \mathrm{kpc}$ ). Figure 5 shows heliocentric distances and sizes in terms of radii defined above. GC are characterized by sizes $O(10)-O(100) \mathrm{pc}$, while galaxies show a larger variation because we include objects of different types, ranging from dwarfs with radius $\sim O(10)$ pc to large galaxies such as Andromeda with major semi-axis $\sim 23 \mathrm{kpc}$. Most galaxies reach distances further than our HVS selection.

Further details about the selected objects are given in appendix C.

\section{METHODOLOGY}

\subsection{Trajectories}

Orbits are integrated using the gala software library (PriceWhelan 2017) ${ }^{6}$. We use its default Milky Way potential model based on four components. Below $G$ indicates the gravitational constant, $r_{s}$ is the radial distance in Galactocentric spherical coordinates, $r_{c}$ and $z_{c}$ are the radial distance and height, respectively, in Galactocentric cylindrical coordinates. The nucleus and bulge follow a Hernquist potential for a spheroid (Hernquist 1990):

$\phi\left(r_{s}\right)=-\frac{G m}{r_{s}+a}$.

The disk follows a Miyamoto-Nagai profile (Miyamoto \& Nagai 1975; Bovy 2015):

$\phi\left(r_{c}, z_{c}\right)=-\frac{G m}{\sqrt{r_{c}^{2}+\left(a+\sqrt{z_{c}^{2}+b^{2}}\right)^{2}}}$.

The halo follows a spherical Navarro-Frenk-White (NFW) profile: (Navarro et al. 1996).

$\phi\left(r_{s}\right)=-\frac{G m}{r_{s}} \ln \left(1+\frac{r_{s}}{a}\right)$.

We use the same parameters as in Marchetti et al. (2019), summarized in table 1 . In the PBH CDM scenario the inner part of the halo is better described by an Einasto profile (Einasto 1965), but this only affects the dynamics close to the Galactic center, where a few of our HVS candidates lie. We have verified that taking into account a power-law gravitational potential profile (Evans 1993, 1994; Calcino et al. 2018) rather than NFW does not affect our conclusions.

We trace trajectories back in time by $100 \mathrm{Myr}$ and $1 \mathrm{Gyr}$ when looking for correlations between HVS and GC or galaxies, respectively, with a resolution of $1 \mathrm{kyr}$ (necessary to resolve the smallest GC). In the case of large and distant objects (Andromeda, LMC and SMC) we use a poorer time resolution of $100 \mathrm{kyr}$, but integrate up to $5 \mathrm{Gyr}$ back in time (to assure that the slower stars in our selection have the time to reach Andromeda distance). ${ }^{7}$

6 https://gala-astro.readthedocs.io

7 More precisely, we integrate orbits setting time steps of $0.1 \mathrm{Myr}$ (10 Myr for Andromeda, LMC and SMC) in gala and then interpolate with cubic splines to reach the desired time resolution. 
Table 1. Milky Way potential parameters.

\begin{tabular}{|c|c|c|}
\hline Component & Potential & Parameters \\
\hline Nucleus & Hernquist & $\begin{array}{l}m=1.71 \times 10^{9} M_{\odot} \\
a=0.07 \mathrm{kpc}\end{array}$ \\
\hline Bulge & Hernquist & $\begin{array}{l}m=5 \times 10^{9} M_{\odot} \\
a=1.0 \mathrm{kpc}\end{array}$ \\
\hline Disk & Miyamoto-Nagai & $\begin{array}{l}m=6.8 \times 10^{10} M_{\odot} \\
a=3 \mathrm{kpc} \\
b=0.28 \mathrm{kpc}\end{array}$ \\
\hline Halo & NFW & $\begin{array}{l}m=5.4 \times 10^{11} M_{\odot} \\
a=15.62 \mathrm{kpc}\end{array}$ \\
\hline
\end{tabular}

\subsection{Impact parameter}

Let $r\left(t_{i}\right)$ be the distance between a star and a GC or galaxy with radius $R$ (defined as discussed in section 2 ) at a given time $t_{i}$. We define the impact parameter for the trajectories of a star and a GC/galaxy as

$\theta \equiv \frac{1}{R} \min _{i} r\left(t_{i}\right)$.

Given the impact parameter likelihood $P(D \mid \theta)$, where $D$ denotes data, we want to identify those stars compatible with having scattered with compact objects bounded to a given GC or galaxy. In the case of GC the radius is determined via proper motion members, and we set $\theta \lesssim 1$ as necessary condition for scattering. In the case of galaxies, the half-light radius (measured along the major axis) or the optical major semi-axis are looser proxies of the underlying DM distribution. Furthermore, dwarf galaxies can have large ellipticity. We take into account these uncertainties by extending the relevant range to $\theta \lesssim 10$ for a star being compatible with having scattered with compact objects bound to a given galaxy, and by studying results as functions of the impact parameter.

\subsection{Likelihoods}

We illustrate how we sample from trajectory parameters space based on observables or derived quantities available in the catalogs described in section 2. Since position errors are dominated by uncertainties on distances, we neglect errors in right-ascension $\alpha$ and declination $\delta$ for computational convenience.

We write the probability distribution for the star trajectory parameters in terms of $\log$-normal ${ }^{8}$ and normal $\mathcal{N}\left(\mu, \sigma^{2}\right)$ distributions $^{9}$

$$
P_{*}=\operatorname{Lognormal}\left(m_{d}, v_{d}\right) \mathcal{N}\left(\boldsymbol{\mu}_{\alpha \beta}, \boldsymbol{\Sigma}_{\mu_{\alpha \beta}}\right) \mathcal{N}\left(V_{r}, \sigma_{V_{r}}^{2}\right) .
$$

Here $d$ is the heliocentric distance discussed in section 2.1 (we find that a log-normal distribution for distances recover

\footnotetext{
8 The Lognormal $\left(m_{x}, v_{x}\right)$ distribution parameters are related to the mean value $x$ and variance $\sigma_{x}^{2}$ of the random variable by $m_{x}=\ln \left(x / \sqrt{1+\sigma_{x}^{2} / x^{2}}\right)$ and $v_{x}=\ln \left(1+\sigma_{x}^{2} / x^{2}\right)$.

9 Some of the data discussed here provides $16 \%$ and $84 \%$ quantiles rather than the variance. We approximate the log-normal or normal distributions variance as the mean of these lower and upper bounds.
}

the respective asymmetric probability distributions), $\boldsymbol{\mu}_{\alpha \beta}=$ $\left(\mu_{\alpha^{*}}, \mu_{\delta}\right)$ with $\Sigma_{\mu_{\alpha \beta}}$ their covariance matrix $\left(\mu_{\alpha^{*}}=\mu_{\alpha} \cos (\delta)\right.$ is proportional to the proper motion in right-ascension direction $\mu_{\alpha}, \mu_{\delta}$ is the proper motion in declination direction), and $V_{r}$ is the radial velocity. Gaia DR2 provides astrometric parameters at epoch J2015.5 that, for comparison with other datasets, we transform to epoch J2000.0 following the reduction procedures used to construct the Hipparcos and Tycho catalogues (ESA 1997) ${ }^{10}$ (more rigorous transformations including the effects of light-travel time are given in Butkevich \& Lindegren (2014), but they are not well suited for negative parallaxes characterizing some of our sources). We verified that the only quantities affected non-negligibly by epoch propagation are $\alpha, \delta$ and for a few sources $V_{r}$.

We write the probability distribution for globular clusters as

$P_{g c}=\mathcal{N}\left(\boldsymbol{X}, \boldsymbol{\Sigma}_{\boldsymbol{X}}\right) \mathcal{N}\left(\boldsymbol{\mu}_{\alpha \beta}, \boldsymbol{\Sigma}_{\mu_{\alpha \beta}}\right) \mathcal{N}\left(V_{r}, \sigma_{V_{r}}^{2}\right)$,

where $\boldsymbol{X}$ are heliocentric Cartesian coordinates used to compute distances. In the case of galaxies we have

$$
\begin{aligned}
P_{\text {gal }}= & \mathcal{N}\left(\mu, \sigma_{\mu}^{2}\right) \mathcal{N}\left(\boldsymbol{\mu}_{\alpha \beta}, \boldsymbol{\Sigma}_{\mu_{\alpha \beta}}\right) \mathcal{N}\left(V_{r}, \sigma_{V_{r}}^{2}\right) \times \\
& \operatorname{Lognormal}\left(m_{R}, v_{R}\right) .
\end{aligned}
$$

Here the heliocentric distance is computed from the distance modulus $\mu$ (for Antlia II we use the derived distance obtained in Torrealba et al. (2019)). For Andromeda we sample directly from the derived Galactocentric velocity error distributions (van der Marel et al. 2012) rather than proper motions and radial velocity. The log-normal distribution for the radius $R$ takes into account the asymmetric error bounds for some of the objects, and the fact that its expectation value is restricted to be positive (we verified that results do not change if we assume a normal distribution and a prior $R>0)$. In the case of Andromeda, LMC, SMC and of GC we don't have information about the respective radius probability distributions, but this is not critical information since afterwards we study results as a function of the impact parameter $\theta \propto 1 / R$.

The impact parameter likelihood $P(D \mid \theta)$ for every star and GC or galaxy pair is obtained by sampling from $P_{*} P_{g c}$ or $P_{*} P_{\text {gal }}$, respectively. We reconstruct each likelihood drawing at least 1000 random samples, necessary to recover Bayes factors at the $O(1 \%)$ level. ${ }^{11}$ We verified that uncertainties in the Galactocentric frame definition, see appendix B, are negligible for our purposes. ${ }^{12}$

We verified that for the cases of our interest $(\theta \lesssim 10)$ likelihoods are well fit by skew log-normal distributions ${ }^{13}$

$P(D \mid \theta)=\frac{1}{\left(\theta-\lambda_{D}\right) \sigma_{D} \sqrt{2 \pi}} \exp \left[-\frac{\left(\ln \left(\theta-\lambda_{D}\right)-\mu_{D}\right)^{2}}{2 \sigma_{D}^{2}}\right]$,

10 We use epoch propagation functions provided by TOPCAT (Taylor 2005).

11 While the sampling can be in principle parallelized over each star and GC/galaxy pair, we are limited by high memory costs to reconstruct only a few likelihoods at the time. This prevents us from running a Markov chain Monte Carlo sampler for each case.

12 For consistency we point out that for the cases shown in section 4 figures we do sample also from uncertainties in the Galactocentric frame.

13 While this form is not well suited for $\theta \rightarrow 0$, we find it reliable down to our smallest sampled values $\theta \sim O\left(10^{-2}\right)$. 
where the fit parameters $\mu_{D}, \sigma_{D}, \lambda_{D}$ are determined for each star and galaxy/GC pair.

Together with the impact parameter likelihoods, we also reconstruct the likelihoods for the scattering time (i.e., the time corresponding to the minimum distance between trajectories).

\subsection{Hypothesis testing}

Given an impact parameter likelihood and a prior $\Pi(\theta)$, the posterior distribution is given by Bayes' theorem $P(\theta \mid D) \propto$ $P(D \mid \theta) \Pi(\theta)$. We can establish at what credible interval a star is compatible with having scattered with compact objects bound to the given system if values $\theta \lesssim O(1)$ (for GC) or $\theta \lesssim O(10)$ (galaxies) are included in the region of interest. Below we consider Bayesian hypothesis testing.

We want to compute the Bayes factor for the hypothesis $H$ that the star trajectory intersects the given galaxy/GC trajectory, relative to the hypothesis $\bar{H}$ of no intersection. Positive evidence for $H$ suggests that the star is compatible with having scattered with compact objects bounded to the given galaxy/GC (possibly fixing a lower threshold for $\theta$ ).

Given the likelihood $P(D \mid \theta)$ computed in section 3.3, the marginal likelihoods under the two hypothesis are:

$$
\begin{aligned}
& P(D \mid H)=\int_{0}^{\infty} \mathrm{d} \theta P(D \mid \theta) \Pi(\theta \mid H), \\
& P(D \mid \bar{H})=1-P(D \mid H) .
\end{aligned}
$$

$\Pi(\theta \mid H)$ models our prior knowledge on the impact parameter distribution under the trajectories intersection hypothesis. We opt for a flat prior

$\Pi(\theta \mid H)= \begin{cases}\frac{1}{\theta_{*}-\theta_{0}} & \theta_{0} \leq \theta \leq \theta_{*} \\ 0 & \text { otherwise. }\end{cases}$

Finally, the Bayes factor is defined by:

$K\left(\theta_{0}, \theta_{*}\right) \equiv \frac{P(D \mid H)}{P(D \mid \bar{H})}$.

We fix the lower prior threshold $\theta_{0}$ at the smallest distance from the GC/galaxy center at which we expect scattering with compact objects. Note that in the PBH CDM scenario, due to the finite size of black holes, the DM density distribution, rather than peaking around a central cusp, may be small in the innermost regions of the galaxy due to the gravitational slingshot effect (García-Bellido 2017), and reach a maximum at a finite distance from the center. We then study the Bayes factor as a function of the upper threshold $\theta_{*}$. In other words, we marginalize over the uncertainties outlined in section 3.2.

Our prior choice is dictated by simplicity given that here we aim at investigating at once several objects with different mass distributions (in some case highly irregular) dependent not only on astrophysical details associated to a given object, but also on the intrinsic nature of DM. Follow-up analyses focused on individual objects should consider priors based on realistic modeling for ejection location and velocity inside a GC/galaxy. We stress that being the problem inherently Bayesian (we have at our disposal only one physical realization of the sources under consideration), discussion cannot disregard a prior choice.
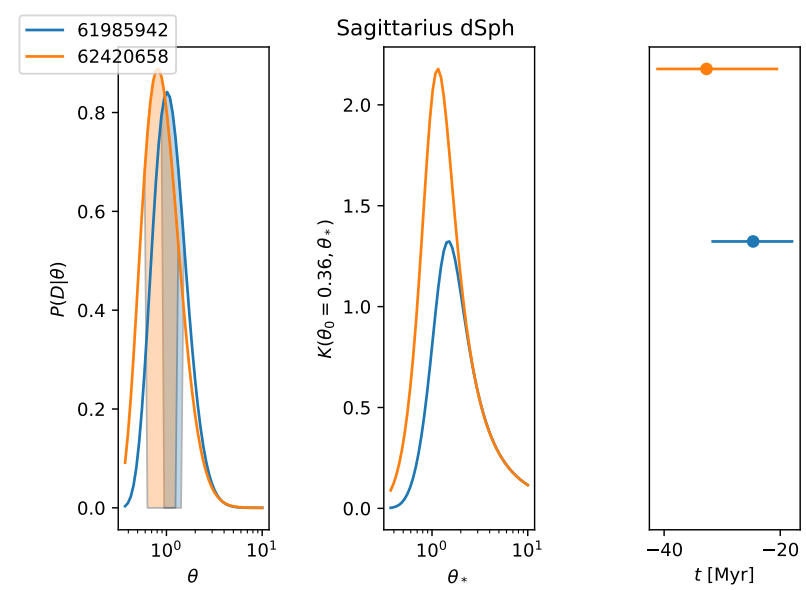

Figure 6. Stars compatible with having scattered with compact objects within Sagittarius dSph assuming Marchetti et al. (2019) distances. The legend shows the first 8 digits of Gaia DR2 star identification numbers. Left panel: Impact parameter likelihood. Filled regions are $68 \%$ confidence intervals. Central panel: Bayes factor for $\theta_{0}=0.36$ as a function of the upper prior threshold $\theta_{*}$. Right panel: Mean and $68 \%$ intervals for the intersection time (today corresponds to $t=0 \mathrm{yr}$ ). The height of error bars is proportional to the respective maximum Bayes factor.

Given the flat prior, the marginal likelihood can be written in terms of the likelihood cumulative distribution function (CDF). While we could compute the empirical CDF, it is numerically convenient to use the analytical form obtained by fitting eq. (8), and obtain an analytical expression for the marginal likelihood

$$
P(D \mid H)=\frac{F\left(\theta_{*}\right)-F\left(\theta_{0}\right)}{\theta_{*}-\theta_{0}},
$$

where $F(x)$ is the CDF up to a given threshold $x$, given in terms of the CDF for the standard normal distribution $\Phi$

$$
\begin{aligned}
F(x) & =\Phi\left(\frac{\ln \left(x-\lambda_{D}\right)-\mu_{D}}{\sigma_{D}}\right) \\
& =\frac{1}{2}\left[1+\operatorname{erf}\left(\frac{\ln \left(x-\lambda_{D}\right)-\mu_{D}}{\sigma_{D} \sqrt{2}}\right)\right],
\end{aligned}
$$

and erf is the error function. We verified that differences in Bayes factors computed using the empirical CDF and the analytical approximation are of order $10 \%$ for likelihoods peaked in the $\theta$ range of interest, and within $5 \%$ for those objects with $K \gtrsim 1$

\section{RESULTS}

We first fix the prior lower threshold $\theta_{0}=0$ and search for scattering events within a given upper threshold $\theta_{*}$. Then we repeat the search for a few values of the lower prior threshold $\theta_{0} \leq 0.5$. This parameterise our belief that a scattering event is unlikely to take place in the innermost region of a given object, as it is the case if we look for interactions with the PBH CDM halo of a galaxy. We consider both Marchetti et al. (2019) and Anders et al. (2019) distance estimates. If 


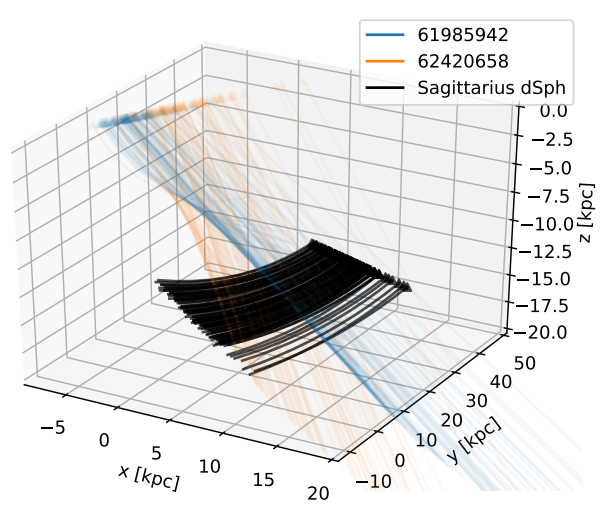

Figure 7. Orbits in Galactocentric Cartesian coordinates corresponding the same objects discussed in figure 6. Line density is proportional to the likelihood. Markers represent positions today.

$K>1$ then the star is compatible with having interacted with a given object.

In the case $\theta_{0}=0$ we do not find candidate scattering events (see however appendix A). As shown in figure 6, in the case of the Sagittarius dwarf spheroidal galaxy (Sagittarius dSph), we find two stars candidate to having scattered with compact objects within it when assuming Marchetti et al. (2019) distances for $\theta_{0}=0.36$, corresponding to the minorto-major axis ratio for Sagittarius $\mathrm{dSph}$ given its ellipticity $\epsilon=0.64 \pm 0.02$ (McConnachie 2012). The Bayes factor peaks at values $\theta_{*} \sim 1$. Scattering times are about 20-40 Myr ago, excluding that these events are directly related to the fact that Sagittarius dSph may have crossed the Galactic disk 300-900 Myr ago (Antoja et al. 2018).

Figure 7 shows 100 orbits randomly sampled from the respective likelihoods for objects discussed in figure 6 . Distance uncertainties lead to large spreads in radial directions from the Sun. Trajectories beyond the scattering event are no longer reliable as they may be depend on a different potential, but that's not a concern for our purposes.

In order to verify whether our results are compatible with the evolutionary status of our stars (i.e., whether their life time is enough to have crossed the very large distance from the original point), we derive their effective temperature $\left(\mathrm{T}_{\mathrm{eff}}\right)$ and bolometric luminosity $\left(\mathrm{L}_{\mathrm{bol}}\right)$ and compare with theoretical isochrones and evolutionary tracks. First, we obtain $\mathrm{L}_{\text {bol }}$ and $\mathrm{T}_{\text {eff }}$ using the Virtual Observatory SED Analyzer (VOSA, Bayo et al. 2008), by constructing a complete Spectral Energy Distribution (SED) taking advantage of different photometric repositories under Virtual Observatory (VO) protocols. Then, the basic properties are derived using atmospheric models by Kurucz (Castelli et al. 1997). We impose restrictions on the surface gravity $(\log g=2)$ and metallicity $([\mathrm{Fe} / \mathrm{H}]=-0.5)$, based on the expectations about these candidates (they should be giant or subgiant stars with low metallicity, since Sagittarius dSph has $[\mathrm{Fe} / \mathrm{H}]=-0.40 \pm 0.2 \mathrm{dex}$ (McConnachie 2012)). In any case, SED fitting depends weakly on $\log g$ and our results are very similar if a larger degree of freedom is allowed.
Thus, the $\mathrm{T}_{\text {eff }}$ range between 6000 and $4250 \mathrm{~K}$, whereas the $\mathrm{L}_{\mathrm{bol}}$ are bracketed between 620 and $2000 \mathrm{~L}_{\odot}$. We have compared these values with PADOVA models (Marigo et al. 2017). Their position in a HR diagram clearly shows that they have masses in the range 3.5-6 M $\odot$ and they are either in the subgiant branch, close to the subgiant branch, or the Blue loop. Therefore, we can establish a lower limit for the age, about $100 \mathrm{Myr}$, fully compatible with our expectations.

Positive evidence is not confirmed when using Anders et al. (2019) distances. However, our result provides motivation to model a prior based on the actual three-dimensional DM distribution of Sagittarius dSph, a difficult issue that has to take into account strong tidal disruption.

More information about the sources discussed here is given in table $\mathrm{C} 4$.

\section{CONCLUSIONS}

We have defined a Bayesian framework to search for correlations between 1642 Gaia DR2 high-velocity star trajectories and those of 52 globular clusters (identified by Gaia DR2) and 35 Milky Way dwarf and nearby galaxies. We report 2 stars candidate to have scattered with compact objects within Sagittarius dSph roughly between 20 and $40 \mathrm{Myr}$ ago when assuming distances estimated in Marchetti et al. (2019). Analysis of their evolutionary status leads to a lower bound of about 100 Myr for their age, fully compatible with the scattering time window. Results are not confirmed when assuming distances estimated in Anders et al. (2019).

These events may correspond to DM scattering, if the latter is composed by $\mathrm{PBH}$ able to accelerate significantly a star upon encounter. In principle the reported number of scattering events may be used to validate this hypothesis. However, given the statistically small sample considered here and uncertainties in PBH mass distribution we cannot put meaningful limits.

Marchetti et al. (2019) reported HVS candidates compatible with extragalactic origin. Their trajectory may also be explained if they scattered with DM bounded to a galaxy or GC. We repeated our search including their final HVS selection of 20 stars (with probability of being unbound larger than $80 \%$ ), and we do not find evidence to support this hypothesis (furthermore several stars in their selection are contaminated by spurious radial velocities (Boubert et al. 2019)).

In defining the impact parameter based on the radius of a sphere centered around a given galaxy or GC, we have taken into account the necessity to analyze at once several heterogeneous objects. Studying the Bayes factor as a function of the marginal likelihood prior takes into account that the radius is only a proxy to the actual shape (e.g., Sagittarius dSph is characterized by a large ellipticity) or DM distribution (that can extend well beyond the optical size). Nevertheless, a follow-up analysis focused on Sagittarius dSph should define the impact parameter based on its actual shape and DM distribution, taking into account its time evolution.

Our results are highly dependent on the distance computation methodology. We find that Marchetti et al. (2019) distances are usually significantly larger than other catalogs for our stellar selection (see table C4). Distances estimated in Anders et al. (2019) are more in agreement with other 
computations (e.g., (Bailer-Jones et al. 2018) and Schönrich et al. (2019)) although results generally differ significantly for stars more distant than $3 \mathrm{kpc}$. This sensitivity on systematic effects (especially a global parallax zero-point difficult to model) calls for future confirmations based on more accurate parallax estimates.

Marchetti et al. (2018) showed that the majority of HVS expected to be detected by Gaia are fainter than the limiting magnitude to obtain radial velocities in DR2. Future data releases will include a larger number of stars with full phase-space information, together with improved astronomy and photometry. As our analysis shows, the understanding of systematic errors up to distances of around $10 \mathrm{kpc}$ is crucial for a robust search. More accurate data will also help to avoid spurious HVS identification possibly affecting our selection.

The fact that we only find candidate scattering events within Sagittarius dSph, relatively large and close, may be due to a selection effect that can be included in future analyses. In fact, if the events under consideration correspond to DM scattering, then we would expect to be able to detect a similar number of interactions also with other large galaxies such as LMC. It is important to repeat and possibly extend the search when future Gaia data releases will be available.

The main motivation of the present search is that HVS may be correlated to past scattering events with massive compact objects in dwarf galaxies. The same idea prompts another interesting prospect, i.e., using trajectories of HVS compatible with having extragalactic origin as a guide for discovery of faint dwarf galaxies, particularly relevant to extend catalogs of low surface brightness galaxies (Du et al. 2019) and for missions like the MESSIER surveyor (VallsGabaud 2016).

\section{ACKNOWLEDGEMENTS}

We thank Alex Drlica-Wagner and Edward (Rocky) Kolb for discussions, and the anonymous referee for constructive comments.

We acknowledge use of the Hydra cluster at IFTUAM/CSIC (Madrid). This research made use of Astropy, ${ }^{14}$ a community-developed core Python package for Astronomy (Astropy Collaboration et al. 2013, 2018), TOPCAT (Taylor 2005) and STILTS (Taylor 2006). This work has made use of data from the European Space Agency (ESA) mission Gaia (https://www.cosmos.esa.int/gaia), processed by the Gaia Data Processing and Analysis Consortium (DPAC, https://www.cosmos.esa.int/web/gaia/ dpac/consortium). Funding for the DPAC has been provided by national institutions, in particular the institutions participating in the Gaia Multilateral Agreement.

FM and JGB are supported by the Research Project FPA2015-68048-C3-3-P [MINECO-FEDER] and the Centro de Excelencia Severo Ochoa Program SEV-2016-0597. DB is been funded by the Spanish State Research Agency (AEI) Project No.ESP2017-87676-C5-1-R and No. MDM2017-0737 Unidad de Excelencia "María de Maeztu"- Centro de Astrobiología (INTA-CSIC).

14 http://www.astropy.org

\section{REFERENCES}

Adén D., et al., 2009, A\&A, 506, 1147 (arXiv:0908.3489)

Anders F., et al., 2019, A\&A, 628, A94 (arXiv:1904.11302)

Antoja T., et al., 2018, Nature, 561, 360 (arXiv:1804.10196)

Astropy Collaboration et al., 2013, A\&A, 558, A33 (arXiv: 1307.6212)

Astropy Collaboration et al., 2018, AJ, 156, 123 (arXiv: 1801.02634)

Bailer-Jones C. A. L., Rybizki J., Fouesneau M., Mantelet G., Andrae R., 2018, AJ, 156, 58 (arXiv:1804.10121)

Bayo A., Rodrigo C., Barrado Y Navascués D., Solano E., Gutiérrez R., Morales-Calderón M., Allard F., 2008, A\&A, 492, 277 (arXiv:0808.0270)

Bechtol K., et al., 2015, ApJ, 807, 50 (arXiv:1503.02584)

Bellazzini M., Gennari N., Ferraro F. R., Sollima A., 2004, MNRAS, 354, 708 (arXiv: astro-ph/0407444)

Bellazzini M., Gennari N., Ferraro F. R., 2005, MNRAS, 360, 185 (arXiv: astro-ph/0503418)

Belokurov V., et al., 2007, ApJ, 654, 897 (arXiv:astro$\mathrm{ph} / 0608448$ )

Belokurov V., et al., 2009, MNRAS, 397, 1748 (arXiv:0903.0818)

Blaauw A., 1960, MNRAS, 121, 164

Bonaca A., Conroy C., Wetzel A., Hopkins P. F., Kereš D., 2017, ApJ, 845, 101 (arXiv:1704.05463)

Bonanos A. Z., Stanek K. Z., Szentgyorgyi A. H., Sasselov D. D., Bakos G. Á., 2004, AJ, 127, 861 (arXiv:astro-ph/0310477)

Boubert D., et al., 2019, MNRAS, 486, 2618 (arXiv:1901.10460)

Bovy J., 2015, ApJS, 216, 29 (arXiv:1412.3451)

Butkevich A. G., Lindegren L., 2014, A\&A, 570, A62 (arXiv: 1407.4664)

Calcino J., Garcia-Bellido J., Davis T. M., 2018, Mon. Not. Roy. Astron. Soc., 479, 2889 (arXiv:1803.09205)

Carignan C., Beaulieu S., Côté S., Demers S., Mateo M., 1998, AJ, 116, 1690 (arXiv:astro-ph/9807222)

Carrera R., Aparicio A., Martínez-Delgado D., Alonso-García J., 2002, AJ, 123, 3199 (arXiv:astro-ph/0203300)

Castelli F., Gratton R. G., Kurucz R. L., 1997, A\&A, 318, 841

Chen B., et al., 2001, ApJ, 553, 184

Chou M.-Y., et al., 2007, ApJ, 670, 346 (arXiv:astro$\mathrm{ph} / 0605101)$

Clesse S., García-Bellido J., 2017, Phys. Dark Univ., 15, 142 (arXiv: 1603.05234)

Coleman M. G., et al., 2007, ApJ, 668, L43

Dall'Ora M., et al., 2006, ApJ, 653, L109 (arXiv:astro$\mathrm{ph} / 0611285)$

Drlica-Wagner A., et al., 2015, ApJ, 813, 109 (arXiv:1508.03622)

Du W., Cheng C., Wu H., Zhu M., Wang Y., 2019, MNRAS, 483, 1754 (arXiv: 1811.04569)

ESA ed. 1997, The HIPPARCOS and TYCHO catalogues. Astrometric and photometric star catalogues derived from the ESA HIPPARCOS Space Astrometry Mission ESA Special Publication Vol. 1200

Einasto J., 1965, Trudy Astrofizicheskogo Instituta Alma-Ata, 5, 87

Evans N. W., 1993, MNRAS, 260, 191

Evans N. W., 1994, MNRAS, 267, 333

Fritz T. K., Battaglia G., Pawlowski M. S., Kallivayalil N., van der Marel R., Sohn S. T., Brook C., Besla G., 2018, A\&A, 619, A103 (arXiv:1805.00908)

Gaia Collaboration et al., 2016, A\&A, 595, A1 (arXiv: 1609.04153)

Gaia Collaboration et al., 2018a, A\&A, 616, A1 (arXiv: 1804.09365)

Gaia Collaboration et al., 2018b, A\&A, 616, A12 (arXiv: 1804.09381)

García-Bellido J., 2017, J. Phys. Conf. Ser., 840, 012032 (arXiv:1702.08275) 
Gillessen S., Eisenhauer F., Trippe S., Alexand er T., Genzel R., Martins F., Ott T., 2009, ApJ, 692, 1075 (arXiv:0810.4674)

Grcevich J., Putman M. E., 2009, ApJ, 696, 385 (arXiv:0901.4975)

Greco C., et al., 2008, ApJ, 675, L73 (arXiv:0712.2241)

Hattori K., Valluri M., Bell E. F., Roederer I. U., 2018, ApJ, 866, 121 (arXiv: 1805.03194)

Hernquist L., 1990, ApJ, 356, 359

Ibata R. A., Gilmore G., Irwin M. J., 1994, Nature, 370, 194

Ibata R. A., Wyse R. F. G., Gilmore G., Irwin M. J., Suntzeff N. B., 1997, AJ, 113, 634 (arXiv:astro-ph/9612025)

Irwin M., Hatzidimitriou D., 1995, MNRAS, 277, 1354

Kirby E. N., Simon J. D., Geha M., Guhathakurta P., Frebel A., 2008, ApJ, 685, L43 (arXiv:0807.1925)

Kirby E. N., Guhathakurta P., Bolte M., Sneden C., Geha M. C., 2009, ApJ, 705, 328 (arXiv:0909.3092)

Kirby E. N., Lanfranchi G. A., Simon J. D., Cohen J. G., Guhathakurta P., 2011, ApJ, 727, 78 (arXiv:1011.4937)

Kirby E. N., Boylan-Kolchin M., Cohen J. G., Geha M., Bullock J. S., Kaplinghat M., 2013, ApJ, 770, 16 (arXiv:1304.6080)

Kirby E. N., Simon J. D., Cohen J. G., 2015, ApJ, 810, 56 (arXiv: 1506.01021)

Koch A., Grebel E. K., Wyse R. F. G., Kleyna J. T., Wilkinson M. I., Harbeck D. R., Gilmore G. F., Evans N. W., 2006, AJ, 131, 895 (arXiv:astro-ph/0511087)

Koch A., et al., 2009, ApJ, 690, 453 (arXiv:0809.0700)

Koposov S. E., et al., 2011, ApJ, 736, 146 (arXiv:1105.4102)

Koposov S. E., Belokurov V., Torrealba G., Evans N. W., 2015, ApJ, 805, 130 (arXiv: 1503.02079)

Laevens B. P. M., et al., 2015a, ApJ, 802, L18 (arXiv: 1503. 05554)

Laevens B. P. M., et al., 2015b, ApJ, 813, 44 (arXiv:1507.07564)

Lee M. G., Yuk I.-S., Park H. S., Harris J., Zaritsky D., 2009, ApJ, 703, 692 (arXiv:0907.5102)

Lützgendorf N., et al., 2012, A\&A, 543, A82 (arXiv:1205.4022)

Majewski S. R., Skrutskie M. F., Weinberg M. D., Ostheimer J. C., 2003, ApJ, 599, 1082 (arXiv:astro-ph/0304198)

Marchetti T., Contigiani O., Rossi E. M., Albert J. G., Brown A. G. A., Sesana A., 2018, MNRAS, 476, 4697 (arXiv: 1711.11397)

Marchetti T., Rossi E. M., Brown A. G. A., 2019, MNRAS, 490, 157 (arXiv: 1804.10607)

Marigo P., et al., 2017, ApJ, 835, 77 (arXiv:1701.08510)

Martin N. F., Ibata R. A., Chapman S. C., Irwin M., Lewis G. F., 2007, MNRAS, 380, 281 (arXiv:0705.4622)

Martin N. F., et al., 2008a, ApJ, 672, L13 (arXiv:0709.3365)

Martin N. F., de Jong J. T. A., Rix H.-W., 2008b, ApJ, 684, 1075 (arXiv:0805.2945)

Martin N. F., et al., 2015, ApJ, 804, L5 (arXiv:1503.06216)

Mateo M., Olszewski E. W., Morrison H. L., 1998, ApJ, 508, L55 (arXiv:astro-ph/9810015)

Mateo M., Olszewski E. W., Walker M. G., 2008, ApJ, 675, 201 (arXiv: 0708.1327)

McConnachie A. W., 2012, AJ, 144, 4 (arXiv:1204.1562)

Miyamoto M., Nagai R., 1975, PASJ, 27, 533

Monaco L., Bellazzini M., Ferraro F. R., Pancino E., 2004, MNRAS, 353, 874 (arXiv: astro-ph/0406350)

Moretti M. I., et al., 2009, ApJ, 699, L125 (arXiv:0906.0700)

Navarro J. F., Frenk C. S., White S. D. M., 1996, Astrophys. J., 462, 563 (arXiv:astro-ph/9508025)

Newton O., Cautun M., Jenkins A., Frenk C. S., Helly J., 2018, Mon. Not. Roy. Astron. Soc., 479, 2853 (arXiv: 1708.04247)

Norris J. E., Wyse R. F. G., Gilmore G., Yong D., Frebel A., Wilkinson M. I., Belokurov V., Zucker D. B., 2010, ApJ, 723, 1632 (arXiv: 1008.0137)

Okamoto S., Arimoto N., Yamada Y., Onodera M., 2008, A\&A, 487, 103 (arXiv: 0804.2976)

Peñarrubia J., et al., 2011, ApJ, 727, L2 (arXiv:1011.6206)

Pietrzyński G., et al., 2008, AJ, 135, 1993 (arXiv:0804.0347)
Pietrzyński G., Górski M., Gieren W., Ivanov V. D., Bresolin F., Kudritzki R.-P., 2009, AJ, 138, 459 (arXiv:0906.0082)

Price-Whelan A. M., 2017, The Journal of Open Source Software, 2,388

Reid M. J., Brunthaler A., 2004, ApJ, 616, 872 (arXiv:astro$\mathrm{ph} / 0408107$ )

Schönrich R., 2012, MNRAS, 427, 274 (arXiv:1207.3079)

Schönrich R., Binney J., Dehnen W., 2010, MNRAS, 403, 1829 (arXiv:0912.3693)

Schönrich R., McMillan P., Eyer L., 2019, MNRAS, 487, 3568 (arXiv: 1902.02355)

Simon J. D., Geha M., 2007, ApJ, 670, 313 (arXiv:0706.0516)

Simon J. D., et al., 2011, ApJ, 733, 46 (arXiv:1007.4198)

Simon J., et al., 2019, BAAS, 51, 409 (arXiv:1903.04743)

Taylor M. B., 2005, in Shopbell P., Britton M., Ebert R., eds, Astronomical Society of the Pacific Conference Series Vol. 347, Astronomical Data Analysis Software and Systems XIV. p. 29

Taylor M. B., 2006, in Gabriel C., Arviset C., Ponz D., Enrique S., eds, Astronomical Society of the Pacific Conference Series Vol. 351, Astronomical Data Analysis Software and Systems XV. p. 666

Torrealba G., et al., 2019, MNRAS, 488, 2743 (arXiv: 1811.04082)

Valls-Gabaud D., 2016. Cambridge University Press, pp 199-201, doi:10.1017/S1743921316011388

Walker M. G., Mateo M., Olszewski E. W., Gnedin O. Y., Wang X., Sen B., Woodroofe M., 2007, ApJ, 667, L53 (arXiv:0708.0010)

Walker M. G., Mateo M., Olszewski E. W., 2008, ApJ, 688, L75 (arXiv:0810.1511)

Walker M. G., Mateo M., Olszewski E. W., 2009a, AJ, 137, 3100 (arXiv:0811.0118)

Walker M. G., Belokurov V., Evans N. W., Irwin M. J., Mateo M., Olszewski E. W., Gilmore G., 2009b, ApJ, 694, L144 (arXiv:0902.3003)

Walker M. G., Mateo M., Olszewski E. W., Peñarrubia J., Evans N. W., Gilmore G., 2009c, ApJ, 704, 1274 (arXiv: 0906.0341)

Walsh S. M., Willman B., Sand D., Harris J., Seth A., Zaritsky D., Jerjen H., 2008, ApJ, 688, 245 (arXiv:0712.3054)

Wenger M., et al., 2000, Astron. Astrophys. Suppl. Ser., 143, 9 (arXiv: astro-ph/0002110)

Wilkinson M. I., Kleyna J. T., Evans N. W., Gilmore G. F., Irwin M. J., Grebel E. K., 2004, ApJ, 611, L21 (arXiv:astro$\mathrm{ph} / 0406520$ )

Willman B., et al., 2006, arXiv e-prints, pp astro-ph/0603486 (arXiv: astro-ph/0603486)

Willman B., Geha M., Strader J., Strigari L. E., Simon J. D., Kirby E., Ho N., Warres A., 2011, AJ, 142, 128 (arXiv: 1007.3499)

Zucker D. B., et al., 2006, ApJ, 650, L41 (arXiv:astro$\mathrm{ph} / 0606633)$

de Jong J. T. A., Martin N. F., Rix H.-W., Smith K. W., Jin S., Macciò A. V., 2010, ApJ, 710, 1664 (arXiv:0912.3251)

van der Marel R. P., Fardal M., Besla G., Beaton R. L., Sohn S. T., Anderson J., Brown T., Guhathakurta P., 2012, ApJ, 753,8 (arXiv: 1205.6864)

\section{APPENDIX A: ALTERNATIVE STELLAR SELECTION}

As an alternative to the stellar selection mentioned in section 2.1, here we consider 1747 stars that still satisfy cuts given in Marchetti et al. (2019), total heliocentric velocities $v-\sigma_{v} \gtrsim 500 \mathrm{~km} / \mathrm{s}$ and exclude possibly contaminated radial velocities (Boubert et al. 2019), but have an higher unbound 

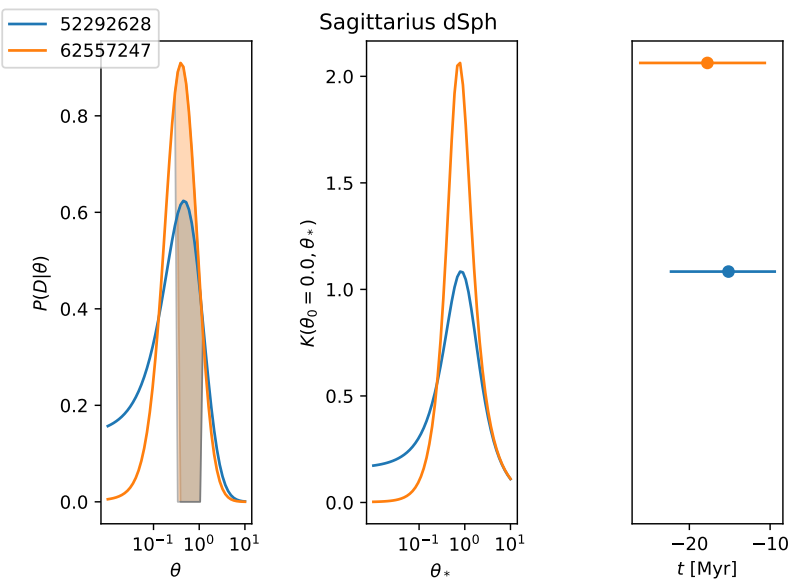

Figure A1. Same as figure 6, but for different stars. The Bayes factor in the central panel is computed setting a lower prior threshold $\theta_{0}=0$.

probability threshold $P_{\mathrm{ub}}>0.9$. Most of the stars have negative parallax, an indicator of poor data quality due to the absence of further photometric and astrometric cuts.

Assuming Marchetti et al. (2019) distances and setting a lower prior threshold $\theta_{0}=0$ for the computation of the Bayes factor, the same search described in section 4 brings to figure A1. Two stars are compatible with having scattered with compact objects in Sagitarius dSph 10-30 Myr ago. If we set $\theta_{0}=0.36$ as discussed in section 4 , we find that the maximum Bayes factors increase to $K \sim 2,10$ for stars 52292628 and 62557247 , respectively, and that a third star (62779130) has positive scattering evidence $(K \gtrsim 1)$. We verified that all stars are compatible with being at least $80 \mathrm{Myr}$ old. (More information about these sources is given in table $\mathrm{C} 4$.)

However, results are not confirmed when we repeate the same search assuming Anders et al. (2019) distances. Furthermore, Anders et al. (2019) flags the aforementioned stars as possible spurious astrometry due to large renormalised unit-weight error (RUWE). Hence, in this case positive evidence for scattering events may be driven not only by distance estimate systematic errors, but also by poor astrometric fit.

\section{APPENDIX B: GALACTOCENTRIC COORDINATES}

Galactocentric coordinates are defined as a Cartesian righthanded system with the $x$-axis pointing from the position of the Sun projected on the Galactic midplane to the Galactic center, the $y$-axis roughly pointing towards Galactic longitude $\ell=90^{\circ}$ and the $z$-axis points roughly towards the North Galactic Pole (we define the Galactic plane to be the normal to the north pole of Galactic coordinates defined by Blaauw (1960)). The Galactic Center right-ascension and declination are taken to be $\alpha=17: 45: 37.224 \mathrm{hr}$ and $\delta=-28: 56: 10.23$ deg, respectively (Reid \& Brunthaler 2004). We assume the distance from the Sun to the Galactic Center to be $8.33 \pm 0.35 \mathrm{kpc}$ (Gillessen et al. 2009) and its height above the
Table C1. Globular clusters identifiers.

\begin{tabular}{lllll}
\hline NGC0104 & NGC5272 & NGC6218 & NGC6388 & NGC6656 \\
NGC0288 & NGC5286 & NGC6254 & NGC6397 & NGC6681 \\
NGC0362 & NGC5466 & NGC6266 & NGC6440 & NGC6752 \\
NGC1851 & NGC5897 & NGC6273 & NGC6453 & NGC6779 \\
NGC1904 & NGC5904 & NGC6284 & NGC6522 & NGC6809 \\
NGC2298 & NGC5986 & NGC6287 & NGC6535 & NGC6838 \\
NGC2808 & NGC6093 & NGC6293 & NGC6541 & NGC6864 \\
NGC3201 & NGC6121 & NGC6304 & NGC6544 & NGC7078 \\
NGC4372 & NGC6144 & NGC6341 & NGC6626 & NGC7089 \\
NGC4833 & NGC6171 & NGC6352 & NGC6637 & NGC7099 \\
NGC5139 & NGC6205 & & & \\
\hline
\end{tabular}

Galactic midplane to be $27 \pm 4$ pc (Chen et al. 2001). Galactocentric velocities are definied assuming a circular velocity of $220 \mathrm{~km} / \mathrm{s}$ at solar radius (Bovy 2015) and a Sun peculiar velocity with respect to the Galactic center $\left(V_{x}, V_{y}, V_{z}\right)_{\odot}=$ $(11.1 \pm 0.74 \pm 1,12.24 \pm 0.47 \pm 2,7.25 \pm 0.37 \pm 0.5) \mathrm{km} / \mathrm{s}$ with additional systematic errors $(1,2,0.5) \mathrm{km} / \mathrm{s}$ (Schönrich et al. 2010).

\section{APPENDIX C: ORBITS DATA}

Tales C1 and C2 list source identifiers for GC and galaxies used in the main analysis. Table C3 shows Sagittarius dSph phase-space data and size. Table C4 shows phase-space data and derived quantities for Gaia DR2 stars compatible with having scattered with compact objects within Sagittarius dSph when assuming Marchetti et al. (2019) distances.

GC, galaxy and star catalogs are available as ancillary files at https://arxiv.org/abs/1907.09298. 
Table C2. Nearby galaxy identifiers and detailed references (complementing those given in section 2.2) for position, size and other useful measurements. All systems are identified as dwarf galaxies, excluded Andromeda, Large Magellanic Cloud (LMC), Small Magellanic Cloud (SMC) and objects of ambiguous nature denoted by an asterisk $(*)$.

\begin{tabular}{|c|c|}
\hline *Draco II & Laevens et al. (2015b) \\
\hline *Grus 1 & Koposov et al. (2015) \\
\hline *Horologium 1 & Koposov et al. (2015); Bechtol et al. (2015) \\
\hline *Reticulum 2 & Bechtol et al. (2015); Koposov et al. (2015) \\
\hline *Triangulum II & Laevens et al. (2015a) \\
\hline *Tucana III & Drlica-Wagner et al. (2015) \\
\hline Andromeda & See section 2.2 \\
\hline Antlia II & See section 2.2 \\
\hline Bootes (I) & $\begin{array}{l}\text { Dall'Ora et al. (2006); Martin et al. (2007, 2008b); Koposov et al. (2011); Grcevich \& Putman } \\
\text { (2009); Norris et al. (2010) }\end{array}$ \\
\hline Bootes II & Walsh et al. (2008); Koch et al. (2009); Martin et al. (2008b); Grcevich \& Putman (2009) \\
\hline Canes Venatici (I) & $\begin{array}{l}\text { Martin et al. (2008a); Simon \& Geha (2007); Martin et al. (2008b); Grcevich \& Putman (2009); } \\
\text { Kirby et al. }(2008,2011)\end{array}$ \\
\hline Canes Venatici II & $\begin{array}{l}\text { Greco et al. (2008); Simon \& Geha (2007); Martin et al. (2008b); Grcevich \& Putman (2009); } \\
\text { Kirby et al. (2008, 2011) }\end{array}$ \\
\hline Carina & $\begin{array}{l}\text { Pietrzyński et al. (2009); Walker et al. (2009a); Irwin \& Hatzidimitriou (1995); Walker et al. } \\
\text { (2008); Grcevich \& Putman (2009); Koch et al. (2006) }\end{array}$ \\
\hline Coma Berenices & $\begin{array}{l}\text { Belokurov et al. (2007); Simon \& Geha (2007); Martin et al. (2008b); Grcevich \& Putman (2009); } \\
\text { Kirby et al. }(2008,2011)\end{array}$ \\
\hline Draco & $\begin{array}{l}\text { Bonanos et al. (2004); Walker et al. (2007); Martin et al. (2008b); Wilkinson et al. (2004); } \\
\text { Grcevich \& Putman (2009); Kirby et al. (2011) }\end{array}$ \\
\hline Eridanus 2 & Koposov et al. (2015); Bechtol et al. (2015) \\
\hline Fornax & $\begin{array}{l}\text { Pietrzyński et al. (2009); Walker et al. (2009a); Irwin \& Hatzidimitriou (1995); Walker et al. } \\
\text { (2008, 2009b); Grcevich \& Putman (2009); Kirby et al. (2011) }\end{array}$ \\
\hline Hercules & $\begin{array}{l}\text { Coleman et al. (2007); Adén et al. (2009); Martin et al. (2008b); Grcevich \& Putman (2009); } \\
\text { Kirby et al. }(2008,2011)\end{array}$ \\
\hline Hydra II & Martin et al. (2015); Kirby et al. (2015) \\
\hline $\mathrm{LMC}$ & See section 2.2 \\
\hline Leo I & $\begin{array}{l}\text { Bellazzini et al. (2004); Mateo et al. (2008); Irwin \& Hatzidimitriou (1995); Grcevich \& Putman } \\
\text { (2009); Kirby et al. (2011) }\end{array}$ \\
\hline Leo II & $\begin{array}{l}\text { Bellazzini et al. (2005); Walker et al. (2007); Irwin \& Hatzidimitriou (1995); Grcevich \& Putman } \\
\text { (2009); Kirby et al. (2011) }\end{array}$ \\
\hline Leo IV & $\begin{array}{l}\text { Moretti et al. (2009); Simon \& Geha (2007); de Jong et al. (2010); Grcevich \& Putman (2009); } \\
\text { Kirby et al. }(2008,2011)\end{array}$ \\
\hline \multicolumn{2}{|l|}{ Leo $\mathrm{V}$} \\
\hline $\mathrm{SMC}$ & See section 2.2 \\
\hline Sagittarius dSph & $\begin{array}{l}\text { Ibata et al. (1994, 1997); Mateo et al. (1998); Majewski et al. (2003); Monaco et al. (2004); } \\
\text { Chou et al. (2007); Grcevich \& Putman (2009); Peñarrubia et al. (2011) }\end{array}$ \\
\hline Sculptor & $\begin{array}{l}\text { Pietrzyński et al. (2008); Walker et al. (2009a); Irwin \& Hatzidimitriou (1995); Walker et al. } \\
\text { (2008); Carignan et al. (1998); Grcevich \& Putman (2009); Kirby et al. (2009, 2011) }\end{array}$ \\
\hline Segue (I) & $\begin{array}{l}\text { Belokurov et al. (2007); Simon et al. (2011); Martin et al. (2008b); Grcevich \& Putman (2009); } \\
\text { Norris et al. (2010) }\end{array}$ \\
\hline Segue II & Belokurov et al. (2009); Kirby et al. (2013) \\
\hline Sextans (I) & $\begin{array}{l}\text { Lee et al. (2009); Walker et al. (2009a); Irwin \& Hatzidimitriou (1995); Walker et al. (2008); } \\
\text { Grcevich \& Putman (2009); Kirby et al. (2011) }\end{array}$ \\
\hline Tucana 2 & Koposov et al. (2015); Bechtol et al. (2015) \\
\hline Ursa Major (I) & $\begin{array}{l}\text { Okamoto et al. (2008); Simon \& Geha (2007); Martin et al. (2008b); Grcevich \& Putman (2009); } \\
\text { Kirby et al. }(2008,2011)\end{array}$ \\
\hline Ursa Major II & $\begin{array}{l}\text { Zucker et al. (2006); Simon \& Geha (2007); Martin et al. (2008b); Grcevich \& Putman (2009); } \\
\text { Kirby et al. }(2008,2011)\end{array}$ \\
\hline Ursa Minor & $\begin{array}{l}\text { Carrera et al. (2002); Walker et al. (2009c); Irwin \& Hatzidimitriou (1995); Wilkinson et al. } \\
\text { (2004); Grcevich \& Putman (2009); Kirby et al. (2011) }\end{array}$ \\
\hline Willman 1 & $\begin{array}{l}\text { Willman et al. (2006); Martin et al. (2007, 2008b); Grcevich \& Putman (2009); Willman et al. } \\
\text { (2011) }\end{array}$ \\
\hline
\end{tabular}




\section{Montanari et al.}

Table C3. Sagittarius dSph phase-space and size data. Rows correspond to right-ascension $\alpha$ and declination $\delta$ (ICRS at epoch J2000.0), distance modulus $\mu$, proper motions in right-ascension direction $\mu_{\alpha *}=\mu_{\alpha} \cos \delta$ and in declination direction $\mu_{\delta}$, radial velocity along the line-of-sight $V_{r}$ and half-light radius measured along the major axis $r_{h}$.

\begin{tabular}{ll}
\hline$\alpha[\mathrm{deg}]$ & 283.8313 \\
$\delta[\mathrm{deg}]$ & -30.4606 \\
$\mu$ & $17.13 \pm 0.11$ \\
$\mu_{\alpha *}[\mathrm{mas} / \mathrm{yr}]$ & $-2.736 \pm 0.044$ \\
$\mu_{\delta}[\mathrm{mas} / \mathrm{yr}]$ & $-1.357 \pm 0.043$ \\
$V_{r}[\mathrm{~km} / \mathrm{s}]$ & $140 \pm 2$ \\
$r_{\boldsymbol{h}}[\operatorname{arcmin}]$ & $342 \pm 12$ \\
\hline
\end{tabular}


Table C4. HVS compatible with having scattered with compact objects within Sagittarius dSph. Columns correspond to Gaia DR2 source identifier, RA and declination, parallax, proper motion in RA direction $\mu_{\alpha *}=\mu_{\alpha *} \cos (\delta)$, proper motion in declination direction, proper motions correlation coefficient, radial velocity and heliocentric distance derived in Marchett et al. (2019), heliocentric distance derived in Anders et al. (2019) and probability of being unbound from the Milky Way potential. The unbound probability relies on Marchetti et al. (2019) distances. Reference epoch for astrometric quantities is J2015.5. While here we report the full source identifier, in the main text we only refer to the first 8 digits. The first two stars are discussed in section 4 , the others in appendix $\mathrm{A}$

\begin{tabular}{|c|c|c|c|c|c|c|c|c|c|}
\hline source & $\begin{array}{c}(\alpha, \delta) \\
{[\mathrm{deg}]}\end{array}$ & $\begin{array}{c}\varpi \\
{[\mathrm{mas}]}\end{array}$ & $\begin{array}{c}\mu_{\alpha *} \\
{[\mathrm{mas} / \mathrm{yr}]}\end{array}$ & $\begin{array}{c}\mu_{\delta} \\
{[\mathrm{mas} / \mathrm{yr}]}\end{array}$ & $c_{\mu_{\alpha *} \mu_{\delta}}$ & $\begin{array}{c}V_{r} \\
{[\mathrm{~km} / \mathrm{s}]}\end{array}$ & $\begin{array}{c}d_{\text {Marchetti }} \\
{[\mathrm{kpc}]}\end{array}$ & $\begin{array}{c}d_{\text {Anders }} \\
{[\mathrm{kpc}]}\end{array}$ & $P_{\mathrm{ub}}$ \\
\hline 6198594250104473728 & $(223.0436,-37.9298)$ & $0.10 \pm 0.04$ & $-21.14 \pm 0.06$ & $2.40 \pm 0.05$ & -0.12 & $96.0 \pm 1.3$ & $9.4_{-2.0}^{+3.3}$ & $5.8_{-1.3}^{+1.9}$ & 0.87 \\
\hline 6242065813132837376 & $(241.4245,-24.0908)$ & $0.08 \pm 0.04$ & $-11.97 \pm 0.07$ & $2.71 \pm 0.04$ & 0.03 & $-60.5 \pm 1.3$ & $11.7_{-3.0}^{+4.8}$ & $7.2_{-1.8}^{+1.8}$ & 0.54 \\
\hline 5229262874909180928 & $(162.8655,-73.4932)$ & $-3.79 \pm 1.14$ & $-30.45 \pm 2.16$ & $38.96 \pm 2.12$ & -0.08 & $51.9 \pm 1.3$ & $8.6_{-3.2}^{+4.8}$ & $2.0_{-0.5}^{+1.8}$ & 0.97 \\
\hline 6255724732546338688 & $(228.2981,-20.7720)$ & $-2.59 \pm 0.73$ & $-31.37 \pm 1.42$ & $10.88 \pm 1.36$ & -0.12 & $-109.3 \pm 0.8$ & $8.6_{-3.6}^{+5.2}$ & $2.3_{-0.8}^{+1: 3}$ & 0.93 \\
\hline 6277913053288720512 & $(214.1732,-20.3474)$ & $-2.02 \pm 0.43$ & $-40.19 \pm 0.69$ & $11.60 \pm 0.54$ & -0.22 & $-39.7 \pm 2.9$ & $10.1_{-3.3}^{+5.5}$ & $4.3_{-1.4}^{+2.8}$ & 1.00 \\
\hline
\end{tabular}




\section{Montanari et al.}

This paper has been typeset from a $\mathrm{T}_{\mathrm{E}} \mathrm{X} / \mathrm{LAT}_{\mathrm{E}} \mathrm{X}$ file prepared by the author. 